\title{
Recyclage et valorisation de sédiments fins de dragage à usage de matériaux routiers
}

\author{
Daniel Levacher*¹, David Colin**, Ana Carina Perroni*2, \\ Zhibo Duan ${ }^{*}$ et Lina Sun $*^{4}$
}

\author{
*Professeur ${ }^{1}$, Ingénieur ${ }^{2}$, Doctorant ${ }^{3}$,Etudiante en Master ${ }^{4}$,Groupe de \\ recherche en Génie Civil, UMR 6143 CNRS, Université de Caen, 14 \\ rue des Tilleuls, F-14000 Caen, zhibo.duan@unicaen.fr \\ **Ingénieur, Docteur, Ets LE FOLL, F-27 Corneville-sur-Risle.
}

\section{Résumé :}

Des alternatives au rejet en mer ou au stockage à terre des sédiments fins ont abouti à la proposition de filières de valorisation. Toute valorisation en tant que matériau routier doit satisfaire les exigences du GTR mais aussi tenir compte de critères économiques. Ainsi, une formulation a été élaborée pour une application en technique routière pour un sédiment de Seine. Le cheminement de la mise au point de cette formulation est présenté et les performances mécaniques discutées. Puis, des essais de faisabilité ont été opérés sur d'autres sédiments pour étudier l'influence des conditions de maturation et de l'ajout de sable fin et la présence des matières organiques. L'analyse des résultats obtenus montre que la formulation reste sensible à la nature des constituants des sédiments de dragage.

Mots-clés : Sédiment de dragage, valorisation, matériau routier, stabilisation

\begin{abstract}
:
Dredging materials pose problems for harbour and river planners, which are more and more confronted with the environmental recommendations. To face with the important volumes of dredged sludge, then the management of these materials arises in terms of storage, treatment and valorisation. A cement-based solidification of dredged sludge with additives is proposed for sediments of Seine River in road construction in respect with GTR rules. The development of a formulation is detailed and discussed with the obtained mechanical performances. A simple formulation is used for other marine sediments in order to evaluate the influence of the curing conditions taking into account the absence of fine sand as additive and the influence of organic matters. Results are reported in the paper and illustrate clearly the importance of the nature of constituents of sediments.
\end{abstract}

Keywords: Dredged sludge, stabilisation/solidification, road material

\section{Contexte}

La raréfaction de sites de dépôts à terre comme en mer associée à une réglementation environnementale de plus en plus contraignante a amené les gestionnaires des ports et du littoral à étudier non seulement le devenir mais aussi 
la valorisation des sédiments marins dragués. La gestion des stocks de sédiments ainsi extraits constitue une véritable problématique environnementale. Les sédiments marins contiennent différents types de contaminants qui, d'une part peuvent selon le degré de contamination et la nature même de ceux-ci être interdits de clapage en mer et d'autre part, être potentiellement " problématiques » dans un procédé de valorisation. Les dragages d'entretien ou d'approfondissement de chenaux génèrent un volume annuel considérable qui oscille entre 50 et 60 $\mathrm{Mm}^{3}$ pour les ports maritimes et d'estuaires français. L'essentiel de ces sédiments révèle un taux de contamination pour lequel il est possible de les claper en mer ou de les stocker à terre. Notre intérêt s'est porté sur la mise au point d'un procédé de stabilisation/solidification des sédiments marins peu ou non pollués pour une valorisation en technique routière. En effet, cette filière peut offrir les avantages suivants : ré-emploi de volumes importants de sédiments traités, substitution aux granulats traditionnels (manque de granulats), alors que certaines se limitent à des tris, à des séparations, à des composts ou à des déchets calcinés pour les sédiments très contaminés. Le traitement étudié est à base de ciment et additifs : chaux et sable fin pour le développement de la formulation de Colin (2003) appliquée à un sédiment de Seine (Port de Rouen) pour des sous couches routières. Une formulation a certes permis de répondre aux exigences requises par le GTR (1992, 2000) mais aussi aux critères économiques en limitant les dosages en ciment et additifs. S'ensuivent deux études d'applicabilité de cette formulation à d'autres sédiments : Calais, Cherbourg, Toulon dont les objectifs étaient de tester non seulement la robustesse de celle-ci mais d'apprécier la sensibilité aux conditions de maturation (méthodologie de préparation) et à la présence de constituants qui associés à des liants spécifiques sont susceptibles d'inhiber ou de perturber la prise. Il est présenté ici d'une part l'influence du mode de conservation des sédiments traités et d'autre part le rôle des matières organiques sur l'amélioration des performances mécaniques.

\section{Le devenir potentiel des sédiments de dragage}

En général, les filières de traitement des sédiments de dragage dépendent de multiples critères indissociables : $i$ - critères économiques (coût économique acceptable), $i i$ - critères techniques (meilleure technique disponible), iii- critères environnementaux (meilleure pratique environnementale), et $i v$ - critères réglementaires (conformité réglementaire). Les filières actuelles les plus répandues sont le dépôt à terre (remplissage de carrières aux CSD...) et l'immersion en mer pour essentiellement les dragages maritimes (clapage) du fait de la simplicité de des procédés techniques utilisés. Dans cette étude, la filière de traitement des sédiments inclut leur valorisation. Alors, le matériau passe d'un simple déchet à éliminer à une ressource naturelle à valoriser.

Sur la figure 1, est illustrée une analyse du devenir potentiel des sédiments de dragage. Le premier critère retenu dans cette analyse est un critère réglementaire, c'est le niveau de contamination selon la législation en vigueur du pays où les travaux de dragage se déroulent, si les sédiments sont considérés sains ou contaminés. En France, l'arrêté interministériel du 14 juin 2000, définit les niveaux de contamination permis selon le Groupe d'Etude et d'Observation sur le 
Dragage et l'Environnement (J.O.2000) pour l'autorisation de clapage en mer. S'il s'avère impossible de les immerger, alors la filière de destination à terre est gérée par une autre législation. Le deuxième critère proposé est en fait une vérification de la granulométrie des sédiments à traiter. En fait il s'agit de juger de la viabilité de réaliser une séparation sable/vase. Ce critère comporte deux analyses. Une première analyse porte sur le rapport "quantité de sable/quantité de vase ». Un étude de faisabilité économique doit être faite sur les volumes à draguer et sur les quantités de sables et de vases. La deuxième analyse est relative à la dimension des sables. En effet, pour être plus largement réutilisés dans le domaine du génie civil, une taille minimale est à retenir, par exemple 1 à quelques $\mathrm{mm}$.

Un troisième critère intéresse l'étude de la faisabilité des traitements des matériaux de dragage pollués. Les types de traitements possibles sont: biologique, physico-chimique et de stabilisation. Il faut remarquer que pour les sédiments pollués, la stabilisation peut être un type de valorisation, comme par exemple l'inertage thermique qui peut générer des produits comme les briques, les graviers artificiels, ...Le dernier critère proposé est d'importance, c'est l'analyse économique des filières de valorisation. Une étude de faisabilité économique et technique doit être menée pour évaluer les techniques de valorisation et ses résultats. L'utilisation préférentielle sera déterminée par le meilleur ratio coût/efficacité et la mise en dépôt ne doit pas être écartée, principalement pour les sédiments très pollués.

\section{Traitement d'un sédiment avec apport d'un correcteur granulométrique}

L'apport d'un correcteur granulométrique à un matériau traité consiste, la plupart du temps, à améliorer son squelette granulaire afin de réduire ses vides. Cette augmentation de la compacité lui permet ainsi de développer de meilleures performances mécaniques. Les sédiments traités présentent une porosité très importante, de l'ordre de $50 \%$, qui suffit à elle seule pour expliquer les faibles performances mécaniques obtenues. Pour réduire cette porosité, l'ajout d'un correcteur granulométrique aux sédiments peut être envisagé. Dans le cas d'un traitement de solidification d'un sédiment fin de dragage, le correcteur ne va pas seulement modifier la compacité du matériau mais l'ensemble des paramètres. Il joue, tout d'abord, un rôle de diluant en modifiant les paramètres de nature (diminution de l'argilosité et de la teneur en matières organiques) et il modifie également la mise en œuvre du matériau.

\subsection{Exemple de traitement d'une vase de Seine avec correcteur}

Un travail expérimental a été mené sur des sédiments de Seine (annexe1) avec trois correcteurs, (Colin, 2003). Le premier est un sable roulé de granularité $0 / 5$ $\mathrm{mm}$, le second, un grès feldspathique $2 / 4 \mathrm{~mm}$ et enfin le troisième, une quartzite $0 / 2 \mathrm{~mm}$. Les dosages en correcteur (en poids sec) ont été de $30 \%$ pour les trois proposés et de $50 \%$ pour le grès et la quartzite. La formulation de traitement destinée à la solidification choisie est la suivante : $2 \%$ de $\mathrm{CaO}$ et $6 \%$ de CPA/CEM I. 
DEVENIR POTENTIEL DES SEDIMENTS DE DRAGAGE

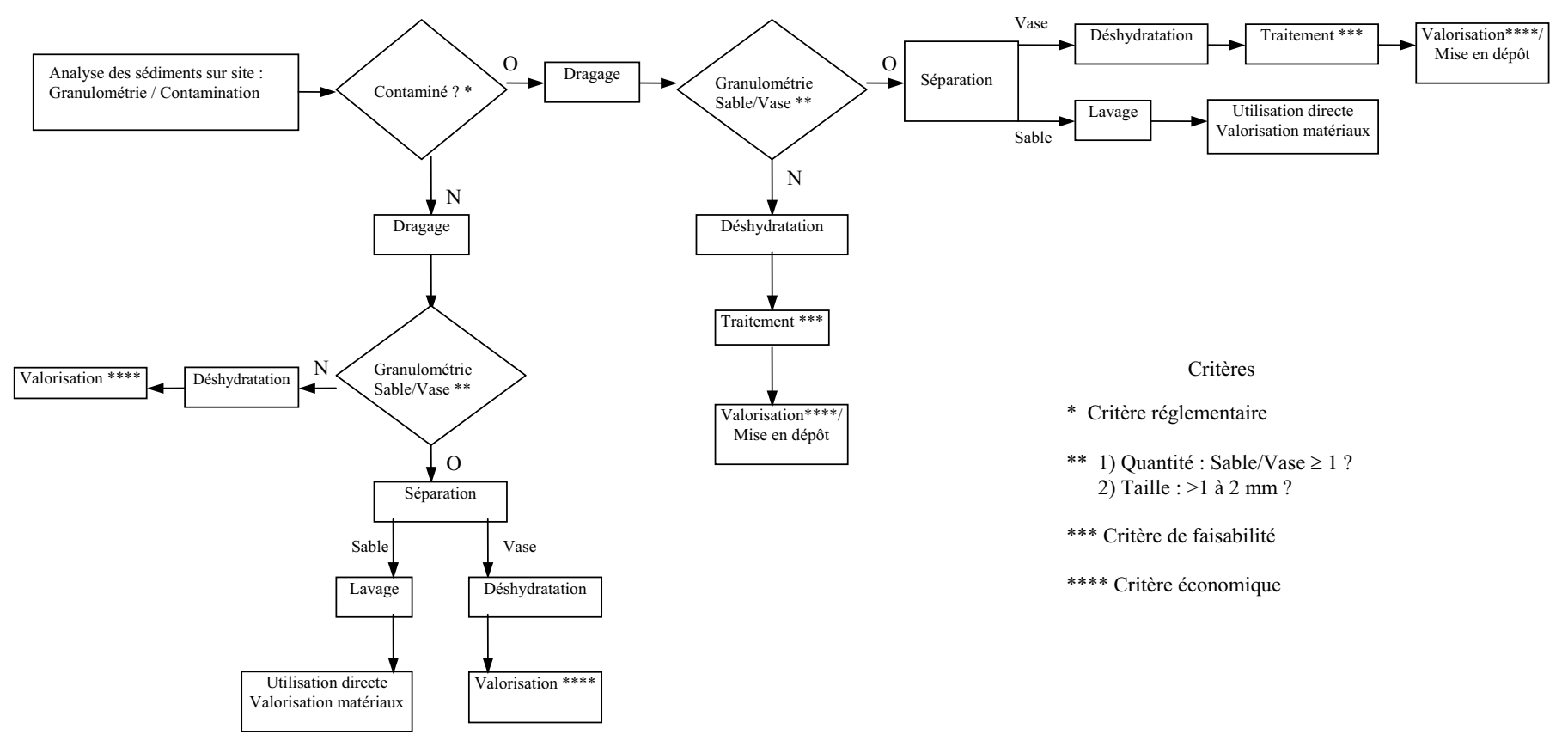

Figure 1. - Chaîne de procédés de traitement possibles et critères retenus 
Le grès apporte uniquement aux sédiments une fraction grossière dont le mode se situe à $4 \mathrm{~mm}$. On se retrouve donc avec un matériau composé essentiellement de fines et de gravillons. Avec la quartzite, c'est au contraire, l'ajout de fines et d'une fraction sableuse. Le pourcentage de fines apportées est de l'ordre de $6 \%$ et de $10 \%$ pour respectivement $30 \%$ et $50 \%$ de quartzite. En ce qui concerne sa fraction sableuse, un premier mode se situe à $0.5 \mathrm{~mm}$ et le reste des grains se trouve entre 1 et $2 \mathrm{~mm}$. Avec le sable roulé, la répartition granulométrique est plus continue et étalée avec un apport de sable (modes à 0.3 et $1.25 \mathrm{~mm}$ ) et d'éléments un peu plus grossiers (mode à $3.15 \mathrm{~mm}$ ).

\subsection{Performances mécaniques à court terme}

Les résistances en compression simple Rc obtenues sont quasiment similaires à celles du matériau traité sans correcteur. A 28 jours, les résistances sont comprises entre 0.5 et $0.8 \mathrm{MPa}$. Le matériau composé de quartzite développe des performances identiques quel que soit le dosage en correcteur. Celui composé de grès, conduit aux performances les plus « extrêmes ». Avec 30\%, les résistances sont les plus «élevées » de toutes avec un $\mathrm{R}_{\mathrm{C}}$ de $0.8 \mathrm{MPa}$ tandis qu'avec $50 \%$, on obtient les résistances les plus faibles $\left(\mathrm{R}_{\mathrm{C}} \approx 0.5 \mathrm{MPa}\right)$. Le matériau composé de grès ne contient pas de fraction intermédiaire entre les fines et les gravillons ce qui doit pénaliser son édifice granulaire. L'existence de plus gros vides dans le matériau avec $50 \%$ de grès pourrait ainsi expliquer des résistances plus faibles. Le sable roulé conduit, quant à lui, à des performances analogues à la quartzite. Une augmentation du dosage en ciment de $2 \%$ a été préconisée. Les éprouvettes ont été moulées en prenant les références de compactage obtenues avec $2 \%$ de $\mathrm{CaO}$ et $6 \%$ de CPA/CEM I et conservées en moule hermétique, mode représentatif de la mise en œuvre des sous couches routières. Les résultats illustrés à la figure 2 , montrent une amélioration des performances pour les matériaux constitués de quartzite.

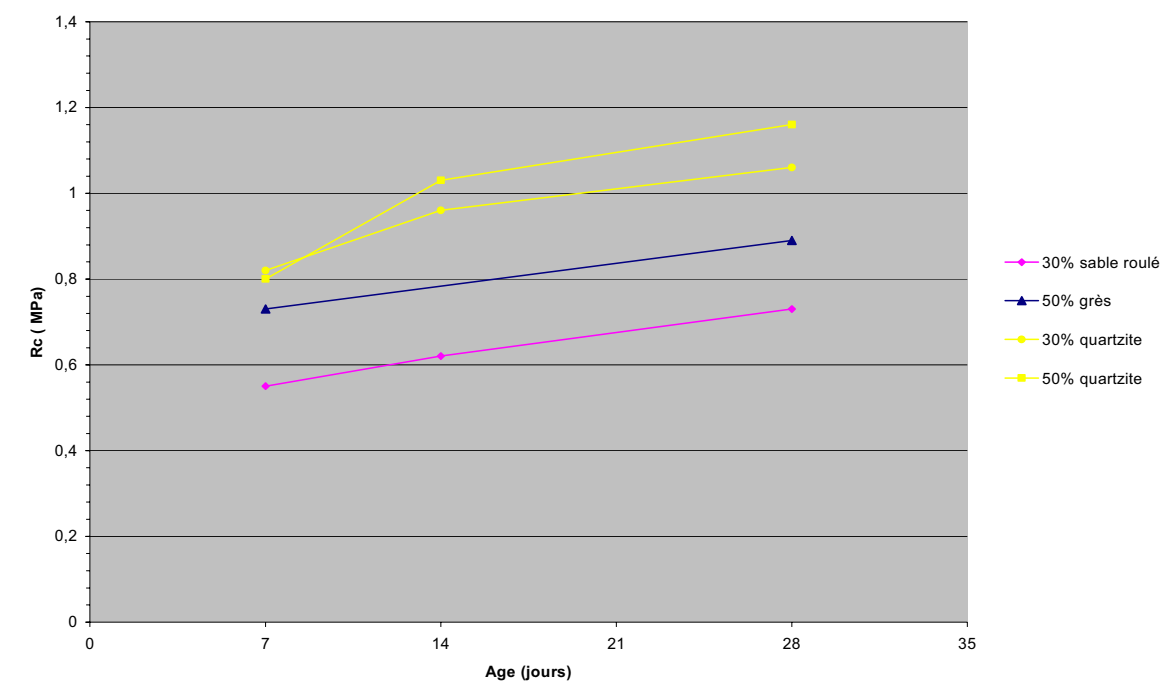

Figure 2. - Performances mécaniques des sédiments avec 30 et $50 \%$ de correcteur traités à $2 \%$ de $\mathrm{CaO}$ et $8 \%$ de $\mathrm{CPA} / \mathrm{CEM}$ I 
Les résistances développées à 28 jours sont, en effet, supérieures à 1.0 MPa. Comme pour la formulation précédente, les deux dosages en quartzite conduisent à des performances semblables. Le matériau composé de sable roulé développe les mêmes résistances qu'avec $6 \%$ de ciment $\left(\mathrm{R}_{\mathrm{C}}=0.7 \mathrm{MPa}\right)$. Pour le matériau à base de grès, il aurait été sans aucun doute plus judicieux de traiter celui avec 30\% d'apport et non avec 50\%. En tout cas, ses performances ne sont pas si faibles puisque l'on obtient à 28 jours, des résistances de l'ordre de $0.9 \mathrm{MPa}$.

\subsection{Discussion sur les correcteurs}

Des trois correcteurs utilisés, seule la quartzite permet de franchir le niveau requis de 1.0 MPa en compression simple et de satisfaire ainsi au critère de traficabilité pour une réutilisation en technique routière. L'utilisation d'un sable fin concassé est donc à privilégier à celles d'un sable roulé et d'un correcteur grossier. Ces résultats sont plutôt logiques. Avec le grès 2/4, il manque certainement une fraction intermédiaire entre les fines et les gravillons ce qui entraîne un mauvais arrangement des grains et par conséquent, la création de gros vides dans le matériau. La nature du sable (concassé ou roulé) influence également les performances du matériau. Par son angularité, la quartzite apporte du frottement aux points de contact entre les grains ce qui n'est pas le cas du sable roulé pour lequel les particules peuvent être assimilées à des billes. Ce frottement inter granulaire permet ainsi au matériau constitué de quartzite de développer de meilleures résistances mécaniques.

\subsection{Proposition de nouvelles formulations}

Deux nouvelles formulations ont été testées. Un traitement mixte $(1 \% \mathrm{CaO} ; 7 \%$ CPA/CEM I) a été mené sur le sédiment de Seine constitué de 30\% de quartzite et un traitement unique au ciment (7\% CPA/CEM I) sur celui réalisé avec $50 \%$ de quartzite.

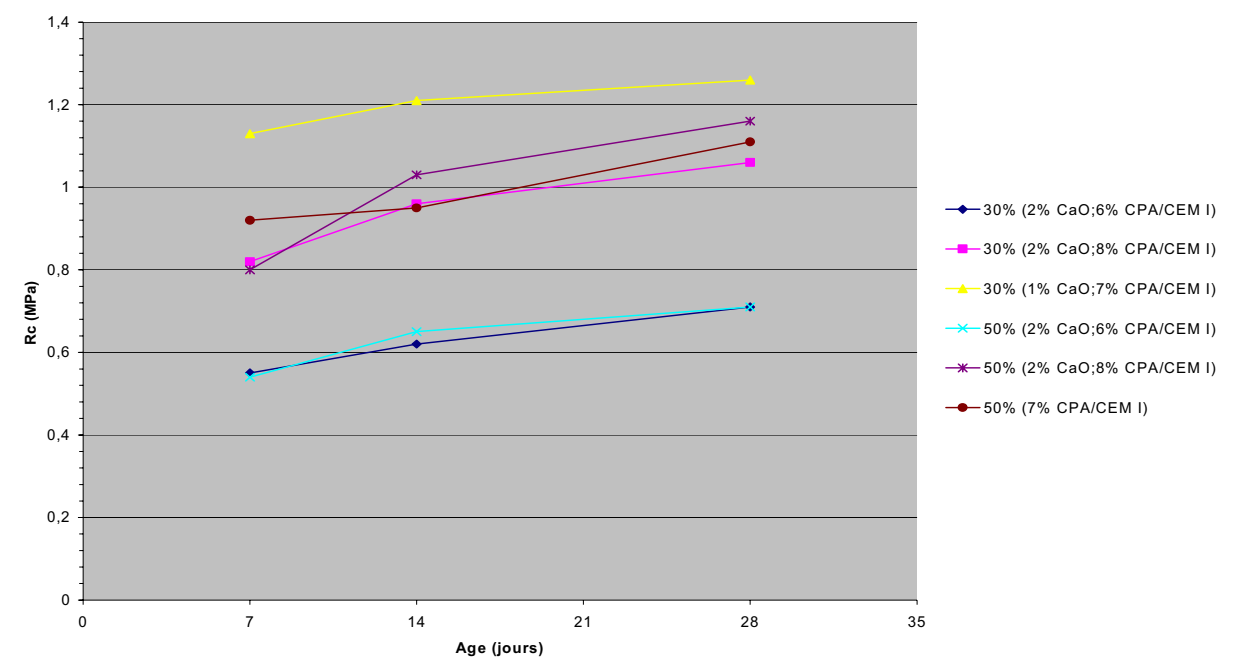

Figure 3. - Performances mécaniques obtenues avec ajout de quartzite pour un ensemble de formulations testées 


\subsubsection{Performances mécaniques à court terme}

La figure 3 présente l'ensemble des résultats obtenus avec ajout de quartzite pour les différents traitements testés. On constate, pour une même formulation, que les performances sont identiques quel que soit le dosage en correcteur apporté. La formulation avec $1 \%$ de $\mathrm{CaO}$ et $7 \%$ de $\mathrm{CPA} / \mathrm{CEM}$ I conduit aux performances les plus élevées avec un $\mathrm{R}_{\mathrm{C}}$ de $1.2 \mathrm{MPa}$ à 28 jours. Un optimum en chaux de $1 \%$ pourrait donc exister. Pour l'expliquer, on peut penser que certaines argiles restent actives et qu'avec juste un pour-cent de chaux, on arrive à les neutraliser.

\subsubsection{Une formulation à retenir appliquée à une vase de Seine}

Les performances mécaniques développées par le matériau composé de $70 \%$ de sédiments et de $30 \%$ de quartzite, même si elles n'atteignent pas un niveau élevé, sont néanmoins suffisantes pour envisager sa valorisation en couche de forme. Le tableau 1 récapitule les performances mécaniques du matériau traité avec la formulation mixte de $1 \%$ de $\mathrm{CaO}$ et $7 \%$ de CPA/CEM I. Soulignons que sa nongélivité demande à être confirmée par un essai de gonflement au gel.

\begin{tabular}{|c|c|c|c|c|c|c|c|}
\hline \multicolumn{3}{|c|}{$\begin{array}{c}\text { Références de mise en } \\
\text { oeuvre }\end{array}$} & \multirow{2}{*}{$\begin{array}{l}\text { Critère de } \\
\text { traficabilité : } \mathbf{R}_{\mathrm{C}} \\
\text { à } 28 \text { jours } \\
(\mathrm{MPa})\end{array}$} & \multirow{2}{*}{$\begin{array}{c}\text { Insensibilité à } \\
\text { l'eau : } \\
\mathbf{R}_{\mathrm{ci}} / \mathbf{R}_{\mathrm{c} 60}\end{array}$} & \multirow{2}{*}{$\begin{array}{c}\text { I. CBR } \\
\text { immersion } \\
(\%)\end{array}$} & \multirow{2}{*}{$\begin{array}{l}\text { Performances à } \\
\text { long terme : } \\
\mathbf{R}_{\mathrm{tb}} \text { à } 90 \text { jours } \\
\text { (MPa) }\end{array}$} & \multirow{2}{*}{$\begin{array}{c}\text { Insensibilité au } \\
\text { gel }\end{array}$} \\
\hline $\begin{array}{c}W_{\text {OPN }} \\
(\%)\end{array}$ & $\begin{array}{c}\rho_{\mathrm{dOPN}} \\
\left(\mathrm{g} / \mathbf{c m}^{3}\right)\end{array}$ & $\begin{array}{l}\text { IPI } \\
(\%)\end{array}$ & & & & & \\
\hline 28 & $1.34-1.38$ & $>10$ & $\geq 1.0$ & $>0.60$ & $40-50$ & $0.16-0.17$ & oui \\
\hline
\end{tabular}

Tableau 1. - Performances mécaniques du matériau composé de $70 \%$ de sédiments et de $30 \%$ de quartzite $0 / 2$ traité avec $1 \%$ de $\mathrm{CaO}$ et $7 \%$ de CPA/CEM I

\section{Autres sédiments de dragage traités avec une formulation sans correcteur}

\subsection{Influence du mode de conservation}

Une formulation simplifiée a été appliquée à d'autres sédiments de dragage, voir tableau 2, en provenance de Calais, Cherbourg et Toulon et à des sédiments de Seine à titre de comparaison. Les échantillons de diamètre $4 \mathrm{~cm}$ et de hauteur $8 \mathrm{~cm}$, pour cette étude ont été confectionnés selon la formulation : $1 \%$ de chaux, $7 \%$ de ciment et $30 \%$ d'eau. La conservation des éprouvettes s'est faite en moules hermétiques et séchage à l'air.

\begin{tabular}{|c|c|c|c|c|}
\hline Origine des sédiments $\rightarrow$ & Calais & Toulon & Seine & Cherbourg \\
\hline Fraction argileuse $(<2 \mu \mathrm{m})$ & $15 \%$ & $10 \%$ & $13 \%$ & $3 \%$ \\
\hline Fraction silteuse $(2 \mathrm{a} 64 \mu \mathrm{m})$ & $85 \%$ & $90 \%$ & $90 \%$ & $70 \%$ \\
\hline Fraction sableuse $(>64 \mu \mathrm{m})$ & 0 & 0 & $7 \%$ & $27 \%$ \\
\hline $\mathrm{d}_{10}(\mu \mathrm{m})$ & 1.2 & 1.8 & 1.4 & 5.2 \\
\hline $\mathrm{d}_{50}(\mu \mathrm{m})$ & 6.7 & 8.6 & 10.4 & 32.5 \\
\hline $\mathrm{d}_{90}(\mu \mathrm{m})$ & 16.7 & 23.0 & 66.9 & 141.3 \\
\hline
\end{tabular}

Tableau 2. - Granulométries des sédiments traités

L'observation des performances mécaniques à court terme montre que le mode de maturation est d'importance. En effet, les résistances à la compression simple Rc 
des éprouvettes conservées dans l'air sont beaucoup plus élevées que celles conservées en moules hermétiques comme le montre la figure 4. Cette influence est à considérer dans la mise en œuvre des matériaux à usage routier.

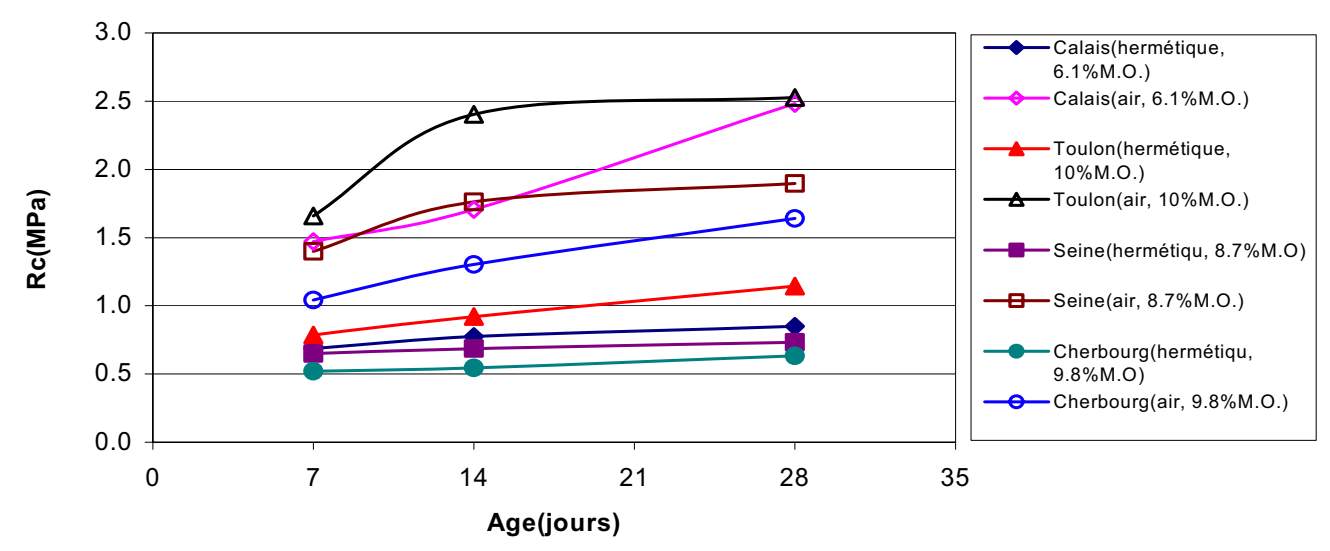

\section{Figure 4. - Evolution des performances mécaniques des sédiments traités}

Les teneurs en eau des éprouvettes stockées à l'air ont été relevées en fonction du temps. Elles sont différentes aux jeunes âges $(<7$ jours) mais elles tendent à 28 jours vers une valeur identique de $8 \%$ pour les 3 sédiments (Calais, Toulon et Seine) qui ont développés à 28 jours les résistances les plus élevées.

\subsection{Rôle d'un ajout de sable fin dans le sédiment}

Il est intéressant de comparer l'incidence d'ajout de sable dans le sédiment traité et conservé en moule hermétique. La figure 5 montre celle-ci pour un sédiment de Seine, (Colin, 2003 ; Sun, 2005), traité avec 1\% de chaux, 7\% de ciment avec ou sans $30 \%$ de quartzite. Ici, le mode de conservation en moule hermétique est représentatif d'une mise en œuvre d'une couche de fondation de chaussée qui est opérée entre 2 couches de liant noir.

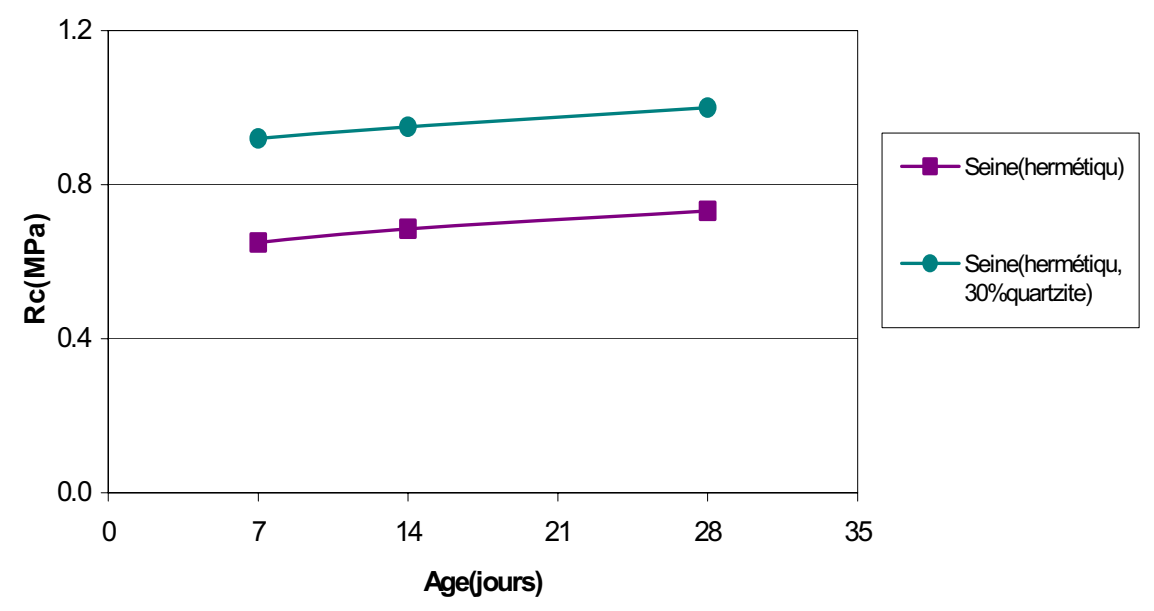

Figure 5. Sédiments de Seine traités avec ajout ou non de sable fin 


\subsection{Rôle des matières organiques dans le traitement des sédiments}

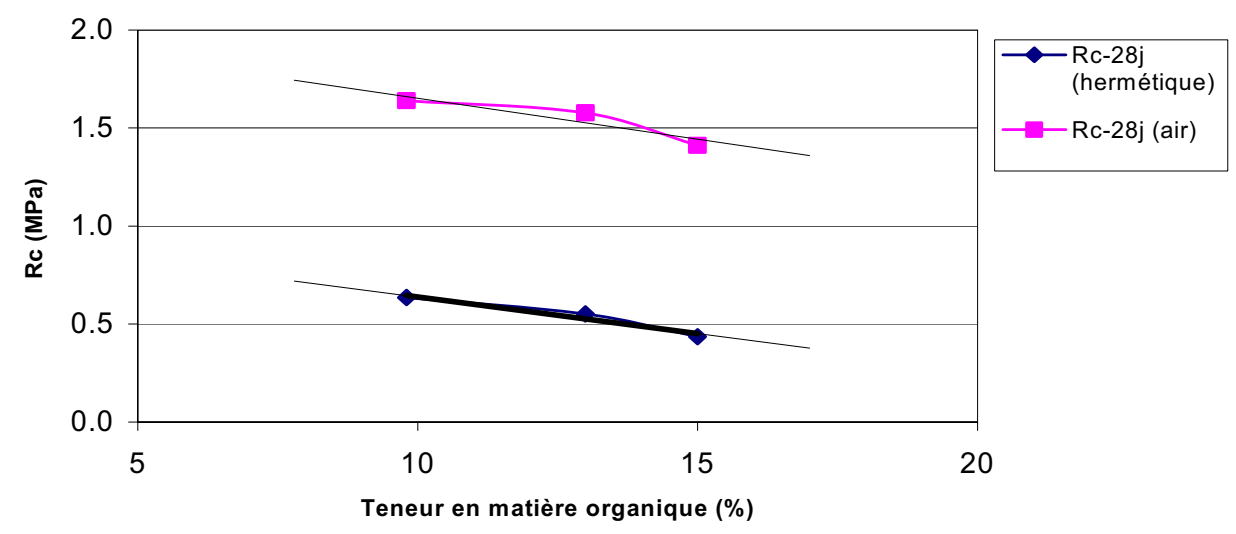

Figure 6. Relation entre les résistances Rc à 28 jours et les matières organiques d'un sédiment traité de Cherbourg.

Un écueil au traitement des sédiments de dragage par solidification à l'aide de ciments et additifs est la présence de matière organique- MO-. Il est intéressant de voir l'évolution des résistances mécaniques en fonction de la teneur en MO. Afin d'étudier le rôle des matières organiques sur le comportement de sédiments traités, des éprouvettes ont été confectionnées avec différents teneurs en matière organique : $9.8 \%$ qui correspond à la teneur en matière organique initiale, $13 \%$ et $15 \%$, (Sun 2005). Le traitement appliqué à un sédiment de dragage de l'avant-port de Cherbourg est identique à celui appliqué aux sédiments de Calais, Toulon et de Seine (§ 4.1.), la méthode de fabrication et le type de conservation sont aussi les mêmes. La figure 6 illustre clairement la diminution des résistances lorsque la teneur en MO augmente quel que soit le mode de conservation des éprouvettes retenu. Les résistances à la compression observées pour ces 3 teneurs en matière organique évoluent ainsi : $\mathrm{Rc}_{9.8 \%}>\mathrm{Rc}_{13 \%}>\mathrm{Rc}_{15 \%}$.

\section{Conclusions et perspectives}

L'objectif d'un traitement de solidification avec correcteur, (Colin, 2003), consistait, à partir d'un sédiment fin dragué près de Rouen, à élaborer un matériau routier susceptible d'être employé pour la réalisation de couches de forme, et puis à partir de formulations testées, les appliquer à d'autres sédiments de dragage

\subsection{Un matériau final valorisable}

$\mathrm{Au}$ terme de ces travaux, l'utilisation de ces sédiments fins en tant que matériau de substitution dans le domaine routier apparaît tout à fait envisageable. La partie expérimentale consacrée à leur traitement a tout d'abord montré que les dosages couramment employés pour le traitement de sols traditionnels du type limon ne permettent pas d'obtenir des performances mécaniques suffisamment élevées. Les travaux ont été orientés vers l'utilisation d'un correcteur granulométrique. Celui- 
ci a tout d'abord un rôle de diluant en diminuant l'argilosité et la teneur en matières organiques. Il modifie également la mise en œuvre du matériau qui se traduit par une diminution de la teneur en eau et une augmentation de la masse volumique à l'OPN. Les différents correcteurs testés ont montré qu'il est préférable d'apporter une fraction fine sableuse concassée à celles d'un sable roulé ou grossier. Grâce notamment à son angularité, la quartzite $0 / 2 \mathrm{~mm}$ apporte $\mathrm{du}$ frottement et permet ainsi de satisfaire au critère de traficabilité et ceci pour une formulation de traitement classique.

\subsection{Un traitement à poursuivre}

Le traitement des sédiments demande à être poursuivi en vue d'une part, d'améliorer les performances mécaniques et d'autre part, de comprendre les phénomènes mis en jeu. On l'a vu, seul le ciment Portland artificiel a permis d'obtenir les performances escomptées. En outre, l'identification précise des composés formant la fraction organique des sédiments demande à être établie. Elle permettra alors de parfaire la connaissance du matériau et d'orienter éventuellement le choix d'un produit de traitement à apporter. L'utilisation d'autres correcteurs pourrait également être envisagée à condition d'intégrer plusieurs paramètres tels que la situation géographique du site de production $d u$ correcteur ainsi que les tonnages produits.

\section{$\underline{\text { 6. Bibliographie }}$}

1 Colin D., (2003), Valorisation des sédiments fins de dragage en technique routière. Thèse de doctorat de l'Université de Caen, 181p.

2 GTR (1992), Guide Technique pour la Réalisation des remblais et des couches de forme. Fascicule I, principes généraux, 100 p.

3 GTS (2000), Traitement des sols à la chaux et aux liants hydrauliques-Guide Technique. LCPC-SETRA, $240 \mathrm{p}$.

4 J.O. (2000), Arrêté du 14 juin 2000 relatif aux niveaux de référence à prendre en compte lors d'une analyse de sédiments marins ou estuariens présents en milieu naturel ou portuaire. JO du 10 août 2000.

5 Sun L., (2005), Traitements des sédiments fins de dragage - Influence des matières organiques sur la stabilisation. Rapport de Master 2 Recherche Génie Côtier, Université de Caen, 50p.

\begin{tabular}{|c|c|}
\hline Granularité & $\begin{array}{l}\text { médiane : } 16.6 \mu \mathrm{m} . \\
\text { passant à } 80 \boldsymbol{\mu m}: 85.5 \% \\
\text { argile }(<\mathbf{2} \boldsymbol{\mu m}): 8.0 \% \\
\text { limon }(\mathbf{2} \text { à } \mathbf{6 3} \boldsymbol{\mu m}): 73.4 \% \\
\text { sable fin }(>\mathbf{6 3} \boldsymbol{\mu m}): 18.6 \%\end{array}$ \\
\hline Teneur en MO (\%) & 7.1 \\
\hline$V B_{S}(g / 100 g)$ & 4.4 \\
\hline $\begin{array}{c}\text { Espèces argileuses (\%) } \\
(\text { pour } 100 \%<2 \mu \mathrm{m})\end{array}$ & $\begin{array}{l}\text { illite : } 20 \\
\text { kaolinite : } 15 \\
\text { smectite : } 65\end{array}$ \\
\hline$\rho_{\mathrm{s}}\left(\mathrm{g} / \mathrm{cm}^{3}\right)$ & 2.56 \\
\hline Classification selon le GTR (1992) & $\mathrm{F}_{11} \mathrm{~A}_{2}$ \\
\hline
\end{tabular}

Annexe 1. - Synthèse sur les caractéristiques des sédiments de Seine traités 